\title{
Spanish and English Vernacular Names of Mammals of North America
}

\author{
Sergio Ticul Álvarez-Castañeda ${ }^{1 *}$ and NoÉ Gónzalez-Ruiz²
}

\begin{abstract}
${ }^{1}$ Centro de Investigaciones Biológicas del Noroeste, Av. Instituto Politécnico Nacional 195, CP. 23096, La Paz. Baja California Sur, México. E-mail: sticul@cibnor.mx (STA-C).

2 Departamento de Biología, División de Ciencias Biológicas y de la Salud, Universidad Autónoma Metropolitana, Unidad Iztapalapa, Apartado Postal 55-535, C.P. 09340. Ciudad de México, México. E-mail: noegr@xanum.uam.mx (NGR).

* Corresponding author
\end{abstract}

\begin{abstract}
Vernacular names are important to know the characteristics of an organism, and are essential to improve the exchange of information between academic and non-academic areas. These are popular in the English language by improving the efficiency of communications in this language, even being more frequently used in the academic environment even when the scientific name is known. In contrast, common names are only occasionally used in Spanish, only for charismatic mammal species; however, these are applied collectively to a wide range of animals that share the same characteristics, such as bats, mice, gophers. Due to the lack of consensus regarding vernacular names in English and of a matching commong name in Spanish for many species, our objective was to gather, rescue and propose vernacular names for 800 species of mammals in North America. The approach used was to follow, to the extent possible, basic principles to propose vernacular names.

Los nombres vernáculos son importantes para conocer las características que tiene un organismo y esenciales para mejorar el intercambio de información entre el ámbito académico y no académico. En el idioma ingles son populares porque representan ventajas al hacer más eficiente la comunicación en su propio idioma, incluso es más utilizado en el medio académico aun cuando se conoce en nombre científico. Pero en el idioma español se usan en ocasiones, solamente son utilizados en especies de mamíferos carismáticas, pero se aplican colectivamente para una amplia gama de animales que comparten las mismas características como los murciélagos, ratones, tuzas. Debido a la falta de consenso de los nombres vernáculos en el idioma inglés y su ausencia en muchas especies en el idioma español, nos dimos a la tarea de recopilar, rescatar y proponer los nombres vernáculos de 800 especies de mamíferos de Norteamérica. Sin embargo, decidimos seguir, en la medida de lo posible, principios básicos para proponer los nombres vernáculos.
\end{abstract}

Keywords: Scientific names; mammals; common name; Mexico.

(C) 2018 Asociación Mexicana de Mastozoología, www.mastozoologiamexicana.org

\section{Introduction}

Since early in history, man has attempted to describe his environment; this required naming the local plants and animals in the surroundings (Clutton-Brock 2015). The names of living organisms serve two essential purposes. The first is that the properties of any organism such as color, size and shape can be associated with its name, so that we can infer the information on an organism through its name alone; for instance, the word "mouse" automatically communicates the intrinsic characteristics of this animal. Second, the name is essential for communication, and the more widespread the name is, the better the communication between people.

These roles of information and communication of names are equally applicable to scientific and vernacular names. However, since the publication of the tenth edition of Sistema Nature (Linnaeus 1758) and after a number of issues of the International Code of Zoological Nomenclature to the latest revision (ICZN 1999), scientific names acquired additional functions, such as being unique and universal, both characteristics not shared by vernacular names. However, the vernacular name is the first thing we know about a mammal (Verts and Carraway 1998); thus, early in life we learn names such as "cat" or "dog". These common names, which we learn from childhood or by interacting and communicating with others in adulthood, are key because they remain in our memory and are part of our common sense. Ultimately, these play an important role in meeting the need to identify and have in place an efficient communication means about a specific kind of mammal (Verts and Carraway 1998).

Another advantage of vernacular names vs. scientific names is that, outside of the academic environment, the latter are little known, unfriendly, and difficult to learn and remember. The lack of knowledge of Greek and Latin roots do not allow to understand and express their meaning to our partners (Álvarez-Castaneda and Alvarez 1996).

In Spanish, vernacular names are little used in scientific literature; in fact, there are questions and discussion as to the definition of common, vulgar and vernacular names (Mones and Mones 1991). Here we define common name as the one used by ordinary people, in most cases at the local level, and generally in little known languages or dialects; this is the case of "citli", a name of Nahuatl origin that refers to the hare (Lepus sp.; Sanchez 1985). Vernacular names are those used in a regional context. These are usually designed by zoologists, and their use has expanded 
over time; an example is the name "murciélago magueyero" (long-nosed bat) mentioned by Arita and Humphrey (1988) to refer to the species of the genus Leptonycteris, the use of which became subsequently widespread. Vulgar name refers to any of the previous two. It must be recognized, however, that in many cases the distinction between these two types of names is ambiguous or unclear. For example, the vulgar names coyote (Canis latrans), ocelot (Leopardus pardalis), and temazate (Mazama sp.) have their origin in the common names of the Nahuatl words "coyotl", "ocelotl", and "temazatl", respectively.

In English, the use of vernacular names is extremely widespread even in the academic environment. It is commonly used in the description of species (e. g., Porter et al. 2017), being used in taxonomic monographs since the end of the 18th century (Kerr 1792; Allen 1894; Merriam 1918), as well as in monographs at the state level in the English language (Hall and Dalquest 1963; Anderson 1972, SánchezHernández et al. 2016). In addition, it is traditionally used in taxonomic list of the mammals of North America (Jones et al. 1973; Hall 1981; Wilson and Ruff 1999; Baker et al. 2003; Reid 2006) and of the world (Wilson and Cole 2000; Wilson and Reeder 2005). U.S. researchers discovered the advantages of the vernacular name, namely that it improves the efficiency of communications by having names in their own language. These names are even more frequently used in the academic environment even when the scientific name is known, and because vernacular names can contribute to resolve taxonomic or nomenclature issues since, in many cases, these are more stable than scientific names (Jackson and Groves 2015).

In Mexico, vernacular names are frequently used in publications to the general public (e. g., Alvarez and González 1987), being widely accepted for birds (Escalante et al. 2014) and marine mammals (Gallo-Reynoso 1985); however, these are rarely used in most scientific publications of terrestrial mammals, even when remarkable reviews are available. Sanchez (1985) performed a search of names in the Mexican Nahuatl dialect, and found 51 names of common use, whereas Gaumer (1917) provided the names of the mammals of Yucatan in the Mayan language; in both cases, common names were correctly associated with the respective scientific names. To the best of our knowledge, pioneering work in Mexico on the use of vernacular names was conducted by Villa (1967) and Ramírez-Pulido et al. (1983). From then on, these have been used in some regional monographs (e. g., Ceballos and Galindo 1984; González-Christen 2010) and in extensive monographs of the mammals of Mexico (Aranda 2000; Villa-Ramirez and Cervantes 2003; Ceballos and Oliva 2005). However, all these publications have particular vernacular names that are unique to charismatic species of mammals, of economic importance in medicine or as food, of in conservation, as is the case of the jaguar (Panthera onca), or the tapir (Tapirella bairdii). Other names are applied to a wide range of mammals that share common characteristics such as bats, mice, and gophers; some of these names are applicable to all species in the same family or belonging to several families. Collective names for similar species are frequently used in many parts of the world (Simpson 1941; Verts and Carraway 1998).

Today, electronic media have made it possible to increase the flow of information and its accessibility. They have also allowed non-specialists in the study of mammals to access information about various species. Vernacular names are particularly important in this case, as they serve as a link between the academia and users that share information: in fact, this is the modern objective of vernacular names (Jackson and Groves 2015). That is why the use of the vernacular names is necessary in disclosure documents or official, as in the Mexican Official Standard 059 (SEMARNAT 2001, 2010) and the Red Book of the International Union for Conservation of Nature and Natural Resources (IUCN; http://www. iucn.org).

We are currently working on keys to identify mammals in North America, where we have recorded 800 species, Based the previous works of Álvarez-Castañeda et al. (2015, 2017). In virtually all cases a vernacular name in English is available for each species, and less than $15 \%$ of species have a name in Spanish. The work will be published as a book in bilingual format; thus, it was decided that all species should have a common name in English and one in Spanish to facilitate their identification. This motivated us to produce a list of names that may be useful and of wide distribution and use at the regional level (Table 1). As vernacular names in English are already extensively used, we only gathered a list, seeking matches between the different lists available, as needed (Jones et al. 1973; Hall 1981; Wilson and Ruff 1999; Wilson and Cole 2000; Baker et al. 2003; Wilson and Reeder 2005; Reid 2006); we designed novel names in the case of either new species or those with recent taxonomic changes. For the Spanish names, we reviewed all the literature to the extent possible (see above), compiling vernacular names commonly used in the literature in Spanish; for the majority of species, however, there is no information on their names, so a common name is proposed for each in these cases.

Formulating vernacular names is complicated and involves many discussions. We are aware that these names are subject to changes, may be potentially misleading, and in some cases may be even absurd and ridiculous (see Mones and Mones 1991). However, as we are convinced of the usefulness of vernacular names (see above), we took the task of collecting, rescuing and proposing vernacular names for all the species of mammals of North America (Table 1). However, to the extent possible, we decided to follow the following basic principles to propose vernacular names, which have been partially discussed by Jackson and Groves (2015):

1) We keep the names that are mentioned frequently in publications and/or that are used in many regions by 
the local people. These apply to the medium-sized and large mammals, such as the jaguarondi (Herpailurus yagouaroundi), puma (Puma concolor), spider monkey (Ateles geoffroyi), etc. Others should be modified as a result of the recent taxonomic changes, for example, the name mantled howler monkey (Alouatta palliata) to differentiate it from the black howler monkey (Alouatta villosa).

2) Vernacular names must serve as a descriptor of the species; thus, they should be informative and facilitate using the name regardless of the extent of knowledge about the species. Accordingly, names are based on characteristics that provide information to the user; this may be a widely used name, a morphological trait, a distinctive coloration, or refer to the geographical distribution of the species. There are groups of names that refer to geographic location, as in the case of the Zempoaltepec Deer Mouse (Peromyscus melanocarpus), Cozumel Harvest Mouse (Reithrodontomys raviventris), or Michoacan Pocket Gopher (Zygogeomys trichopus); others highlight a unique feature that differentiates a particular species from the rest: Collared Pika (Ochotona collaris), White-toothed Woodrat (Neotoma leucodon), or Black-eared Rice Rat (Handleyomys melanotis).

3) In both English and Spanish, our aim was that the name indicate the relationship between species; hence, the first (in Spanish) or last (in English) part of the name refers to the genus. For example we use "water mouse" or "ratita nadadora" for the genus Rheomys, and "spiny pocket mouse" or "ratón espinoso" for Heteromys. In both cases, we avoided using the same vernacular name for any other genus of rodent. Separately, it was decided to designate the genus Habromys using the name "ratón arborícola" to maintain the relationship between the species in the genus, although not all species are strictly arboreal (LeonPaniagua et al. 2007).

4) We seek to choose the shortest names possible, although the two prerequisites just mentioned prevented this in some cases. In addition, names are written in singular to make them shorter, as in "Mexican shrew" instead of "Mexican shrews".

5) The vernacular name should be easy to pronounce, be euphonious and hence easy to remember.

6) Vernacular names are written in lower case, except when these include the name of a geographic place or a proper noun.

7) We avoided using translations of vernacular names in English for the respective names in Spanish, as they do not convey sufficient information in the latter, even within the scientific community itself. Some are derived directly from the scientific name, as in the case of Geoffroy's Tailless Bat (Anoura geoffroyi), Findley's Myotis (Myotis findleyi) or Baker's Harvest Mouse (Reithrodontomys bakeri); in other cases, we not used the name of the author who described the species, as in Thomas's Fruit-eating Bat (Dermanura watsoni), Davis's Round-eared Bat (Lophostoma evotis) or Baird's Pocket Gopher (Geomys breviceps). In both cases, most persons are not familiar with the meaning of the name, thus making its use difficult. Adequate translations from English are used in some cases, provided these meet the above criteria; in other cases, completely new names were designed.

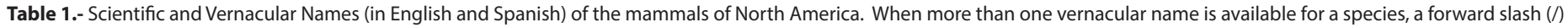
is used to separate each individual name.

\begin{tabular}{|c|c|c|}
\hline Nombre cientifico & Nombre en inglés & Nombre en español \\
\hline \multicolumn{3}{|c|}{ Artiodactyla } \\
\hline \multicolumn{3}{|l|}{ Antilocapridae } \\
\hline Antilocapra americana & Pronghorn & berrendo \\
\hline \multicolumn{3}{|l|}{ Bovidae } \\
\hline Bison bison & American bison & bisonte americano \\
\hline Oreamnos americanus & Mountain goat & cabra de montaña/cabra blanca \\
\hline Ovibos moschatus & Muskox & buey almizclero/toro almizclero \\
\hline Ovis canadensis & Bighorn sheep & borrego cimarrón \\
\hline Ovis dalli & Dall's sheep & $\begin{array}{l}\text { borrego cimarrón de las rocallosas/ } \\
\text { borrego cimarrón blanco }\end{array}$ \\
\hline \multicolumn{3}{|l|}{ Cervidae } \\
\hline Alces americanus & Moose & alce \\
\hline Cervus canadensis & Wapiti & wapití/ciervo canadiense \\
\hline Cervus elaphus & Elk/Red deer & ciervo rojo \\
\hline Mazama pandora & Yucatan brown brocket & temazate café/yuk café, mazate, cabrito café \\
\hline Mazama temama & Central american red brocket & temazate rojo/yuk rojo, mazate rojo, cabrito rojo \\
\hline Odocoileus hemionus & Mule deer & venado bura \\
\hline Odocoileus virginianus & White-tailed deer & venado cola blanca \\
\hline Rangifer tarandus & Caribou/Reindeer & reno/caribú \\
\hline \multicolumn{3}{|l|}{ Tayassuidae } \\
\hline Dicotyles angulatus & Western collared peccary & jabalí de collar del oeste \\
\hline
\end{tabular}

\begin{tabular}{|c|c|c|}
\hline Dicotyles crassus & Eastern collared peccary & pecarí o jabalí de collar del este \\
\hline Tayassu pecari & White-lipped peccary & pecarí o jabalí de labios blancos, senso \\
\hline & Carnive & \\
\hline Canidae & & \\
\hline Canis latrans & Coyote & coyote \\
\hline Canis lupus & Gray wolf & lobo gris, lobo de bosque \\
\hline Canis lycaon & Eastern wolf & lobo canadiense \\
\hline Canis rufus & Red wolf & lobo rojo \\
\hline Urocyon cinereoargenteus & Gray fox & zorra gris \\
\hline Urocyon littoralis & Island gray fox & zorra gris de isla catalina \\
\hline Vulpes lagopus & Arctic fox & zorra del ártico \\
\hline Vulpes macrotis & Kit fox & zorra del desierto, zorra norteña \\
\hline Vulpes velox & Swift fox & zorra del desierto \\
\hline Vulpes vulpes & Red fox & zorro rojo \\
\hline Felidae & & \\
\hline Leopardus pardalis & Ocelot & ocelote \\
\hline Leopardus wiedii & Margay & tigrillo/margay \\
\hline Lynx canadensis & Canada lynx & lince canadiense \\
\hline Lynx rufus & Bobcat & gato montés/lince rojo/lince americano \\
\hline Panthera onca & Jaguar & jaguar/tigre/pantera \\
\hline Puma concolor & Cougar & puma/león, león de montaña \\
\hline Herpailurus yagouaroundi & Jaguarundi & Jaguarundi/leoncillo/onza real \\
\hline
\end{tabular}


Continue...

\section{Mephitidae}

Conepatus leuconotus

Conepatus semistriatus

Mephitis macroura

Mephitis mephitis

Spilogale angustifrons

Spilogale gracilis

Spilogale putorius

Spilogale pygmaea

Eira barbara

Enhydra lutris

Galictis vittato

Gulo gulo

Lontra canadensis

Lontra longicaudis

Martes americana

Martes pennanti

Mustela erminea

Mustela frenata

Mustela nigripes

Mustela nivalis

Neovison vison

Taxidea taxus

Odobenidae

Odobenus rosmarus

\section{Otaridae}

Arctocephalus philippii

Callorhinus ursinus

Eumetopias jubatus

Zalopus californicus

\section{Phocidae}

Cystophora cristata

Erignathus barbatus

Halichoerus grypus

Histriophoca fasciata

Mirounga angustirostris

Monachus tropicalis

Pagophilus groenlandicus

Phoca largha

Phoca vitulina

Pusa hispida

\section{Procyonidae}

Bassariscus astutus

Bassariscus sumichrasti

Nasua narica

Potos flavus

Procyon lotor

Procyon pygmaeus

\section{Ursidae}

Ursus americanus

Ursus arctos

Ursus maritimus

\section{Balaenidae}

Balaena mysticetus

Eubalaenajaponica
American hog-nosed skunk

Striped hog-nosed skunk

Hooded skunk

Striped skunk

Southern spotted skunk

Western spotted skunk

Eastern spotted skunk

Pygmy spotted skunk

Tayra

Sea otter

Greater grison

Wolverine

North american river otter

Neotropical otter

American marten

Fisher

Ermine

Long-tailed weasel

Black-footed ferret

Least weasel

American mink

American badger

Walrus

Guadalupe fur seal

Northern fur seal

Steller sea lion

California sea lion

Hooded seal

Bearded seal

Gray seal

Ribbon seal

Northern elephant seal

Caribbean monk seal

Harp seal

Spotted seal

Harbor seal

Ringed seal

Ringtail

Cacomistle

White-nosed coati

Kinkajou

Raccoon

Cozumel raccoon

American black bear

Brown bear, grizzly bear

Polar bear

Bowhead

North Pacific Right Whale zorrillo espalda blanca

zorrillo bilistado

zorrillo rayado sureño

zorrillo rayado norteño

zorrillo manchado del sur

zorrillo manchado del oeste

zorrillo manchado del este

zorrillo pigmeo

tayra/cabeza de viejo/viejo de monte

nutria marina

grisón

glotón/carcayú

nutria de río norteamericana

nutria de río/perro de agua

marta americana

marta pescadora

armiño

comadreja, oncita, hurón

hurón de patas negras

comadreja pequeña

mink

tlalcoyote/tejón

morsa

lobo fino de guadalupe

lobo marino del ártico

león marino del norte

león marino de California

foca capuchina

foca barbuda

foca gris

foca listada

elefante marino

foca del caribe

foca pia

foca manchada

foca común/foca moteada

foca anillada

cacomixtle/sietillo/babisuri

cacomixtle tropical/huiloncha

coatí/pizote/amátze/tejón

martucha/mico de noche

mapache

mapache pigmeo

oso negro

oso pardo/grizzly

oso polar

ballena de Groenlandia/ballena boreal

ballena franca
Balaenopteridae

\begin{tabular}{|c|c|c|}
\hline Balaenoptera acutorostrata & Common Minke Whale & rorcual enano/rorcual picudo \\
\hline Balaenoptera borealis & Sei Whale & rorcual boreal \\
\hline Balaenoptera edeni & Bryde's Whale & rorcual tropical \\
\hline Balaenoptera musculus & Blue Whale & ballena azul/rorcual azul \\
\hline Balaenoptera physalus & Fin Whale & rorcual común/rorcual de aleta \\
\hline Megaptera novaeangliae & Humpback Whale & ballena jorobada/rorcual jorobado \\
\hline
\end{tabular}

\section{Eschrichtiidae}

Eschrichtius robustus Gray Whale ballena gris

\section{Delphinidae}

Delphinus capensis Long-beaked Common Dolphin delfín común de rostro largo

Delphinus delphis Short-beaked Common Dolphin delfín común

Feresa attenuata Pygmy Killer Whale orca pigmea

Globicephalamacrorhynchus Short-finned Pilot Whale calderón/ballena piloto

Globicephala melas Long-finned Pilot Whale

Grampus griseus

Risso's Dolphin

ballena piloto de aleta larga/calderón común

Lagenodelphis hosei Fraser's Dolphin delfín de botella

Lagenorhynchus acutus Atlantic White-sided Dolphin

Lagenorhynchus albirostris White-beaked Dolphin

delfín de flancos blancos

Lagenorhynchus obliquidens Pacific White-sided Dolphin delfín de costados blancos

Lissodelphis borealis

Northern Right Whale Dolphin

Orcinus orca

Peponocephala electra

Killer Whale

delfín liso borea

Pseudorca crassidens

Melon-headed Whale

Stenella attenuata

False Killer Whale

ballena cabeza de melón

Pantropical Spotted Dolphin

falsa orca/orca bastarda

Stenella clymene

Clymene Dolphin

delfín manchado tropical

Stenella coeruleoalba

Striped Dolphin

Stenella frontalis

Atlantic Spotted Dolphin

Stenella longirostris

Spinner Dolphin

Steno bredanensis

Rough-toothed Dolphin

Tursiops truncatus

Bottlenose Dolphin

delfín giradora del atlántico

delfín rayado

delfín manchado del Atlántico

delfín tornillo

delfín de dientes rugosos

Monodontidae

Delphinapterus leucas

Monodon monoceros

Beluga

Narwhal

beluga

Phocoenidae

Phocoena sinus

Phocoenoides dalli

Vaquita

Dall's Porpoise

\section{Physeteridae}

Kogia breviceps

Kogia sima

Physeter macrocephalus

Pygmy Sperm Whale

Dwarf Sperm Whale

Sperm Whale

\section{Ziphiidae}

Berardius bairdii

Indopacetus pacificus

Baird's Beaked Whale

Tropical Bottlenose Whale

Mesoplodon bidens

Mesoplodon carlhubbsi

Mesoplodon densirostris

Mesoplodon europaeus

Mesoplodon ginkgodens

Mesoplodon mirus

Mesoplodon peruvianus

Ziphius cavirostris

Sowerby's Beaked Whale

Hubb's Beaked Whale

Blainville's Beaked Whale

Gervais's Beaked Whale

Ginkgo-toothed Beaked Whale

True's Beaked Whale

Pygmy Beaked Whale

Cuvier's Beaked Whale

\section{Chiroptera}

Emballonuridae

Balantiopteryx io

Balantiopteryx plicata
Thomas's sac-winged bat

Gray sac-winged bat murciélago de sacos gris murciélago de sacos pardo

Continue... 
Continue...

Centronycteris centralis

Diclidurus albus

Peropteryx kappleri

Peropteryx macrotis

Rhynchonycteris naso

Saccopteryx bilineata

Saccopteryx leptura

Molossidae

Cynomops mexicanus

Eumops auripendulus

Eumops ferox

Eumops floridanus

Eumops hansae

Eumops nanus

Eumops perotis

Eumops underwoodi

Molossus alvarezi

Molossus aztecus

Molossus coibensis

Molossus molossus

Molossus rufus

Molossus sinaloae

Nyctinomops aurispinosus

Nyctinomops femorosaccus

Nyctinomops laticaudatus

Nyctinomops macrotis

Promops centralis

Tadarida brasiliensis

Mormoopidae

Mormoops megalophylla

Pteronotus davyi

Pteronotus gymnonotus

Pteronotus parnellii

Pteronotus personatus

Natalidae

Natalus lanatus

Natalus mexicanus

Noctilionidae

Noctilio albiventris

Noctilio leporinus

Phyllostomidae

Anoura geoffroyi

Artibeus hirsutus

Artibeus jamaicensis

Artibeus lituratus

Dermanura azteca

Dermanura phaeotis

Dermanura tolteca

Dermanura watsoni

Carollia perspicillata

Carollia sowelli

Carollia subrufa

Centurio senex

Chiroderma salvini

Chiroderma villosum

Choeroniscus godmani
Thomas's shaggy bat

Northern ghost bat

Greater dog-like bat

Lesser dog-like bat

Proboscis bat

Greater sac-winged bat

Lesser sac-winged bat

\section{Mexican dog-faced bat}

Black bonneted bat

Central america bonneted bat

Florida bonneted bat

Sanborn's bonneted bat

Eastern lesser bonneted bat

Greater bonneted bat

Underwood's bonneted bat

Alvarez's mastiff bat

Aztec mastiff bat

Coiban mastiff bat

Pallas's mastiff bat

Black mastiff bat

Sinaloan mastiff bat

Peale's free-tailed bat

Pocketed free-tailed bat

Broad-eared free-tailed bat

Big free-tailed bat

Big crested mastiff bat

Brazilian free-tailed bat

Ghost-faced bat

Davy's naked-backed bat

Big naked-backed bat

Common mustached bat

Wagner's mustached bat

Hairy funnel-eared bats

Mexican greater funnel-eared bat

Lesser bulldog bat

Greater bulldog bat

Geoffroy's tailless bat

Hairy fruit-eating bat

Jamaican fruit-eating bat

Great fruit-eating bat

Aztec fruit-eating bat

Pygmy fruit-eating bat

Toltec fruit-eating bat

Thomas's fruit-eating bat

Seba's short-tailed bat

Sowell's short-tailed bat

Gray short-tailed bat

Wrinkle-faced bat

Salvin's big-eyed bat

Hairy big-eyed bat

Godman's long-tongued bat murciélago cara arrugada

murciélago de espalda desnuda menor

murciélago de espalda desnuda mayor

murciélago bigotudo mayor

murciélago bigotudo meno

murciélago orejas de embudo lanudo

murciélago lanudo

murciélago perro mayor

murciélago rayado mayor

murciélago cara de perro mexicano

murciélago de gorra negro

murciélago de gorra de Florida

murciélago de gorra pigmeo

murciélago de gorra del Pacífico

murciélago mastín de Coiban

murciélago mastín peludo

murciélago mastín de Sinaloa

murciélago de cola libre

murciélago de cola libre de bolsa

murciélago de cola libre de orejas grandes

murciélago de cola libre mayor

murciélago mastín crestado

murciélago mexicano de cola libre

murciélago orejas de embudo mexicano

murciélago pescador menor

murciélago pescador mayor

murciélago sin cola gris

murciélago frutero de patas peludas

murciélago frutero común/murciélago zapotero

murciélago frutero gigante

murciélago frutero menor de patas peludas

murciélago frutero pigmeo

murciélago frutero menor de patas desnudas

murciélago frutero Centroamericano

murciélago colicorta

murciélago colicorta pardo

murciélago colicorta gris

murciélago cara de viejo

murciélago ojudo pardo

murciélago ojudo güero

murciélago de cola larga
Choeronycteris mexicana

Chrotopterus auritus

Desmodus rotundus

Diaemus youngi

Diphylla ecaudata

Enchisthenes hartii

Glossophaga commissarisi

Glossophaga leachii

Glossophaga morenoi

Glossophaga soricina

Glyphonycteris sylvestris

Hylonycteris underwoodi

Lampronycteris brachyotis

Leptonycteris nivalis

Leptonycteris yerbabuenae

Lichonycteris obscura

Lonchorhina aurita

Lophostoma brasiliense

Lophostoma evotis

Macrophyllum macrophyllum

Macrotus californicus

Macrotus waterhousii

Micronycteris microtis

Micronycteris schmidtorum

Mimon cozumelae

Mimon crenulatum

Musonycteris harrison

Phylloderma stenops

Phyllostomus discolor

Platyrrhinus helleri

Sturnira hondurensis

Sturnira parvidens

Tonatia saurophila

Trachops cirrhosus

Trinycteris nicefori

Uroderma bilobatum

Uroderma magnirostrum

Vampyressa thyone

Vampyrodes major

Vampyrum spectrum

\section{Thyropteridae}

Thyroptera tricolor

\section{Vespertilionidae}

Antrozous pallidus

Bauerus dubiaquercus

Corynorhinus mexicanus

Corynorhinus rafinesquii

Corynorhinus townsendil

Eptesicus brasiliensis

Eptesicus furinalis

Eptesicus fuscus

Euderma maculatum

Idionycteris phyllotis

Lasionycteris noctivagans

Lasiurus blossevillii

Lasiurus borealis
Mexican long-tongued bat

Woolly False Vampire bat

Common vampire bat

White-winged vampire bat

Hairy-legged vampire bat

Velvety fruit-eating bat

Commissaris's long-tongued bat

Gray's long-tongued bat

Western long-tongued bat

Pallas's long-tongued bat

Tri-colored big-eared bat

Underwood's long-tongued bat

Yellow-throated big-eared bat

Mexican long-nosed bat

Lesser long-nosed bat

Dark long-tongued bat

Common sword-nosed bat

Pygmy round-eared bat

Davis's round-eared bat

Long-legged bat

California big-eared bats

Waterhouse's big-eared bats

Common little big-eared bats

Schmidts's little big-eared bats

Cozumelan golden bat

Striped hairy-nosed bat

Banana bat

Pale-faced bat

Pale spear-nosed bat

Heller's broad-nosed bat

Highland yellow-shouldered bat

Little yellow-shouldered bat

Stripe-headed round-eared bat

Fringe-lipped bat

Niceforo's big-eared bat

Tent-making bat

Brown tent-making bat

Northern little yellow-eared bat

Great stripe-faced bat

Spectral bat

Spix's disk-winged bat

Pallid bat

Van gelder's bat

Mexican big-eared bat

Rafinesque's big-eared ba

Townsend's big-eared bat

Brazilian brown bat

Argentinian brown bat

Big brown bat

Spotted bat

Allen's big-eared bat

Silver-haired bat

Western red bat

Eastern red bat

murciélago trompudo mexicano

falso vampiro lanudo

vampiro común

vampiro de alas blancas

vampiro de patas peludas

murciélago frutero negro

murciélago lenguilargo pequeño

murciélago lenguilargo gris

murciélago lenguilargo del oeste

murciélago lenguilargo común

murciélago tricolor

murciélago lenguilargo moreno

murciélago orejón de garganta amarilla

murciélago magueyero mayor

murciélago magueyero menor

murciélago lenguilargo oscuro

murciélago nariz de espada

murciélago orejas redondas brasileño

murciélago orejas redondas Mesoamericano

murciélago patas largas

murciélago orejón norteño 
Continue..

Lasiurus cinereus

Lasiurus ega

Lasiurus intermedius

Lasiurus seminolus

Lasiurus xanthinus

Myotis albescens

Myotis auriculus

Myotis austroriparius

Myotis californicus

Myotis ciliolabrum

Myotis elegans

Myotis evotis

Myotis findleyi

Myotis fortidens

Myotis grisescens

Myotis keaysi

Myotis keenii

Myotis leibii

Myotis lucifugus

Myotis melanorhinus

Myotis nigricans

Myotis occultus

Myotis pilosatibialis

Myotis planiceps

Myotis septentrionalis

Myotis sodalis

Myotis thysanodes

Myotis velifer

Myotis vivesi

Myotis volans

Myotis yumanensis

Nycticeius humeralis

Parastrellus hesperus

Perimyotis subflavus

Rhogeessa aeneus

Rhogeessa alleni

Rhogeessa bickhami

Rhogeessa genowaysi

Rhogeessa gracilis

Rhogeessa mira

Rhogeessa parvula

Rhogeessa tumida

\section{Dasypodidae}

Cabassous centralis

Dasypus novemcinctus

\section{Didelphidae}

Caluromys derbianus

Chironectes minimus

Didelphis marsupialis

Didelphis virginiana

Marmosa mexicana

Metachirus nudicaudatus

Philander opossum

\section{Northern naked-tailed armadillo armadillo de cola desnuda}

Nine-banded armadillo

DideLPHIOMORPHA

murciélago colapeluda canoso

murciélago colapeluda amarillo del sur

murciélago colapeluda amarillo del norte

murciélago colapeluda canoso de Florida

murciélago colapeluda amarillo del oeste

murciélago ratón plateado

murciélago ratón suroccidental

murciélago ratón del sureste

murciélago ratón de California

murciélago ratón occidental

murciélago ratón tropical/murciélago ratón elegante

murciélago del oeste

murciélago ratón de las Islas Marías

murciélago ratón canela

murciélago ratón gris

murciélago ratón pata peluda

murciélago ratón de la Columbia Británica

murciélago ratón oriental

murciélago ratón Norteamericano

murciélago ratón de nariz negra

murciélago ratón negro

murciélago ratón de Arizona

murciélago ratón patas peludas

murciélago ratón de cabeza plana

murciélago ratón grande del norte

murciélago ratón de Indíana

murciélago ratón peludo

murciélago ratón de las cuevas

murciélago ratón pescador

murciélago ratón patas largas

murciélago ratón pequeño

murciélago del anochecer

murciélago pequeño del oeste

murciélago pequeño del este

murciélago amarillo de Yucatán

murciélago amarillo grande

murciélago amarillo de la costa Chiapas

murciélago amarillo de Chiapas

murciélago amarillo delgado

murciélago amarillo pigmeo

murciélago amarillo pequeño

Little yellow bat

murciélago amarillo de alas negras

\section{Cingulata}

armadillo/armadillo de nueve bandas

Derby's woolly opossum

Water opossum

Common opossum

Virginia opossum

Mexican mouse opossum

Brown four-eyed opossum

Gray four-eyed opossum tlacuache lanudo

tlacuache de agua

tlacuache sureño/zarigüeya sureña

tlacuache norteño/zarigüeya norteña

ratón tlacuache mexicano

tlacuache de cuatro ojos moreno/cuatro ojillos moreno

tlacuache de cuatro ojos gris/cuatro ojillos gris

$\begin{array}{ll}\text { Tlacuatzin balsasensis } & \text { Balsas mouse opossum } \\ \text { Tlacuatzin canescens } & \text { Gray mouse opossum } \\ \text { Tlacuatzin gaumeri } & \text { Yucatan mouse opossum } \\ \text { Tlacuatzin insularis } & \text { Tres Marías mouse oposum } \\ \text { Tlacuatzin sinaloae } & \text { Northern mouse opossum }\end{array}$

tlacuachín/ratón tlacuache del Balsas tlacuachín/ratón tlacuache del Pacífico tlacuachín/ratón tlacuache Yucateco tlacuachín/ratón tlacuache de Tres Marías tlacuachín/ratón tlacuache norteño

LAGOMORPHA

\section{Leporidae}

Brachylagus idahoensis

Lepus alleni

Lepus americanus

Lepus arcticus

Lepus californicus

Lepus callotis

Lepus flavigularis

Lepus othus

Lepus townsendii

Romerolagus diazi

Sylvilagus aquaticus

Sylvilagus auduboni

Sylvilagus bachmani

Sylvilagus gabbi

Sylvilagus cognatus

Sylvilagus cunicularius

Sylvilagus floridanus

Sylvilagus graysoni

Sylvilagus insonus

Sylvilagus nuttallii

Sylvilagus obscurus

Sylvilagus palustris

Sylvilagus robustus

Sylvilagus transitionalis

Ochotonidae

Ochotona collaris

Ochotona princeps

\section{Tapiridae}

Tapirella bairdi

\section{Cyclopedidae}

Cyclopes didactylus

Myrmecophagidae

Tamandua mexicana

Atelidae

\section{Alouatta palliata \\ Alouatta villosa \\ Ateles geoffroyi}

Aplodontidae

Aplodontia rufa

Castoridae

Castor canadensis

Cricetidae

Arborimus albipes

Arborimus longicaudus anteater

Northern tamandua

Pygmy rabbit

Antelope jackrabbit

Snowshoe hare

Arctic hare

Black-tailed jackrabbit

White-sided jackrabbit

Tehuantepec jackrabbit

Alaskan hare

White-tailed jackrabbit

Volcano rabbit

Swamp rabbit

Desert cottontail

Brush rabbit

Tropical cottontail

Manzano mountain cottontail

Mexican cottontail

Eastern cottontail

Tres marias cottontail

Omiltemi cottontail

Mountain cottontail

Appalachian cottontail

Marsh rabbit

Robust cottontail

New england cottontail

Collared pika

American pika

Baird's tapir

tapir centroamericano/danta

PILosa

osito hormiguero dorado

tamandúa/oso hormiguero/brazo fuerte/ chupamiel

Primates

Mantled howler

mono aullador de manto/saraguato de manto

Guatemalan black howler

Geoffroy's spider monkey

mono aullador negro/saraguato negro

mono araña

Rodentia

Sewellel

castor de montaña

American beaver

castor

White-footed vole

chincolo arborícola de patas blancas

Red tree vole 
Continue...

Arborimus pomo

Baiomys musculus

Sonoma tree vole

Baiomys taylori

Southern pygmy mouse

Dicrostonyx groenlandicus Nearctic collared lemming

Dicrostonyx hudsonius

Dicrostonyx nelsoni

Dicrostonyx nunatakensis

Dicrostonyx richardsoni

Dicrostonyx unalascensis

Habromys chinanteco

Habromys delicatulus

Habromys ixtlan

Habromys lepturus

Habromys lophurus

Habromys schmidlyi

Habromys simulatus

Handleyomys alfaroi

Handleyomys chapmani

Handleyomys guerrerensis

Handleyomys melanotis

Handleyomys rhabdops

Handleyomys rostratus

Handleyomys saturatior

Hodomys alleni

Lemmiscus curtatus

Lemmus trimucronatus

Megadontomys cryophilus

Megadontomys nelsoni

Megadontomys thomasi

Microtus abbreviatus

Microtus brewer

Microtus californicus

Microtus canicaudus

Microtus chrotorrhinus

Microtus guatemalensis

Microtus longicaudus

Microtus mexicanus

Microtus miurus

Microtus mogollonensis

Microtus montanus

Microtus oaxacensis

Microtus ochrogaster

Microtus oeconomus

Microtus oregon

Microtus pennsylvanicus

Microtus pinetorum

Microtus quasiater

Microtus richardsoni

Microtus townsendii

Microtus umbrosus

Microtus xanthognathus

Myodes californicus

Myodes gapperi

Myodes rutilus

Nelsonia goldmani

Nelsonia neotomodon

Ungava collared lemming

Nelson's collared lemming

Chinanteco deermouse

Delicate deermouse

Ixtlán deermouse

Zempoaltepec deermouse

Crested-tailed deermouse

Jico deermouse

Alfaro's rice rat

Chapman's rice rat

Guerrero rice rat

Black-eared rice rat

Highland rice rat

Long-nosed rice rat

Cloud forest rice rat

Allen's woodrat

Sagebrush vole

Nearctic brown lemming

Insular vole

Beach vole

California vole

Rock vole

Guatemalan vole

Long-tailed vole

Mexican vole

Singing vole

Mogollon Vole

Tarabundí vole

Prairie vole

Tundra vole

Creeping vole

Meadow vole

Woodland vole

Jalapan vole

North American water vole

Townsend's vole

Zempoaltépec vole

Taiga vole

Western red-backed vole

Southern red-backed vole

Northern red-backed vole chincolo arborícola de Sonoma

ratón pigmeo del su

ratón pigmeo del norte

leming de tundra común

leming de tundra de Ungava

Richardson's collared lemming leming de tundra de la Bahía Hudson

Umnak island collared lemming leming de tundra de la isla Umnak

ratón de arbóreo de Oaxaca

Schmidlyi crested-tailed mouse

ratón de arbóreo delicado

ratón de arbóreo de Ixtlán

ratón de arbóreo de Zempoaltepec

ratón de arbóreo de cola crestada

ratón de arbóreo de Guerrero

ratón de arbóreo Sierra Madre Oriental

ratón de agua tropical

ratón de agua de la Sierra Madre Oriental

ratón de agua de Guerrero

ratón de agua de orejas negras

ratón de agua de las tierras altas

ratón de agua de nariz larga

ratón de agua del bosque de niebla

rata de campo del occidente

chincolo de las artemisas

leming pardo

Oaxacan big-toothed deermouse ratón gigante de Oaxaca

Nelson's big-toothed deermouse ratón gigante de Veracruz

Thomas' big-toothed deermouse ratón gigante de Guerrero

Gray-tailed vole

Montane vole

ratón colicorto/metorito de la Isla San Mateo

ratón colicorto/metorito de la Isla Muskeget

ratón colicorto/metorito de California

ratón colicorto/metorito de cola gris

ratón colicorto/metorito de las rocas

ratón colicorto/metorito de Guatemala

ratón colicorto/metorito de cola larga

ratón colicorto/metorito mexicano común

ratón colicorto/metorito del Yukon

ratón colicorto/metorito Mogollón

ratón colicorto/metorito de las montañas

ratón colicorto/metorito de Oaxaca

ratón colicorto/metorito de las praderas

ratón colicorto/metorito nórdico

ratón colicorto/metorito de Oregon

ratón colicorto/metorito de los pastizales

ratón colicorto/metorito de los bosques

ratón colicorto/metorito de Jalapa

ratón colicorto/metorito de agua

ratón colicorto/metorito de la costa noroeste

ratón colicorto/metorito del cerro Zempoaltepec

ratón colicorto/metorito de la taiga

chincolo de lomo rojo del oeste

chincolo de lomo rojo del sur

chincolo de lomo rojo del norte

Goldman's diminutive woodrat rata enana michoacana

Western diminutive woodrat rata enana del oeste
Neofiber alleni

Neotoma albigula

Neotoma angustapalato

Neotoma bryanti

Neotoma cinerea

Neotoma devia

Neotoma ferruginea

Neotoma floridana

Neotoma fuscipes

Neotoma goldmani

Neotoma insularis

Neotoma lepida

Neotoma leucodon

Neotoma macrotis

Neotoma magister

Neotoma melanura

Neotoma mexicana

Neotoma micropus

Neotoma nelsoni

Neotoma palatina

Neotoma phenax

Neotoma picta

Neotoma stephensi

Neotomodon alstoni

Nyctomys sumichrasti

Ochrotomys nuttalli

Oligoryzomys fulvescens

Ondatra zibethicus

Onychomys arenicola

Onychomys leucogaster

Onychomys torridus

Oryzomys couesi

Oryzomys albiventer

Oryzomys nelsoni

Oryzomys palustris

Oryzomys peninsulae

Oryzomys texensis

Osgoodomys banderanus

Otonyctomys hatti

Ototylomys phyllotis

Ototylomys chiapensis

Peromyscus attwateri

Peromyscus avius

Peromyscus aztecus

Peromyscus beatae

Peromyscus boylii

Peromyscus bullatus

Peromyscus californicus

Peromyscus caniceps

Peromyscus carletoni

Peromyscus crinitus

Peromyscus dickeyi

Peromyscus difficilis

Peromyscus eremicus

Peromyscus eva

Peromyscus fraterculus
Round-tailed muskrat

White-throated woodrat

Tamaulipan woodrat

Bryant's woodrat

Bushy-tailed woodrat

Arizona woodrat

Guatemala woodrat

Eastern woodrat

Dusky-footed woodrat

Goldman's woodrat

Ángel de la Guarda island woodrat rata de campo de Isla Ángel de la Guarda

Desert woodrat

White-toothed woodrat

Big-eared woodrat

Allegheny woodrat

Dark woodrat

Mexican woodrat

Southern plains woodrat

Nelson's woodrat

Bolaños woodrat

Sonoran woodrat

Tehuantepec woodrat

Stephen's woodrat

Volcano deermouse

Sumichrast's vesper rat

Golden mouse

Fulvous colilargo

Common muskrat

Chihuahuan grasshopper mous

Northern grasshopper mouse

Southern grasshopper mouse

Coues's rice rat

White-bellied rice rat

Nelson's rice rat

Marsh rice rat

Lower California rice rat

Texas rice rat

Michoacan deer mouse

Yucatán vesper rat

Big-eared climbing rat

Chiapas big-eared climbing rat

Texas deermouse

Cerralvo island deermouse

Aztec deermouse

Orizaba deermouse

Brush deermouse

Perote deermouse

California deermouse

Monserrat Island deermouse

Carleton's deermouse

Canyon deermouse

Dickey's deermouse

Southern rock deermouse

Cactus deermouse

Southern baja deermouse

Northern baja deermouse

rata almizclera de cola redonda

rata de campo de garganta blanca/rata nopalera

rata de campo de Tamaulipeca

rata de campo de Baja California

rata de campo de cola peluda

ata de campo de Arizona

rata de campo de Guatemala

rata de campo del este

rata de campo de patas negras

rata de campo del altiplano

rata de campo del desierto

rata de campo de las planicies

rata de campo de orejas grandes

rata de campo de los Apalaches

rata de campo negruzca

rata magueyera mexicana

rata de campo de las planicies del sur 
Continue...

Peromyscus furvus

Peromyscus gossypinus

Peromyscus gratus

Peromyscus guardia

Blackish deermouse

Cotton deermouse

Saxicoline deermouse

La Guarda deermouse

Peromyscus guatemalensis Guatemalan deermouse

Peromyscus gymnotis

Peromyscus hooperi

Peromyscus hylocetes

Peromyscus interparietalis

Peromyscus keen

Peromyscus laceianus

Peromyscus latirostris

Peromyscus leucopus

Peromyscus levipes

Peromyscus madrensis

Peromyscus maniculatus

Peromyscus megalops

Peromyscus mekisturus

Peromyscus melanocarpus

Peromyscus melanophrys

Peromyscus melanotis

Peromyscus melanurus

Peromyscus merriami

Peromyscus mexicanus

Peromyscus nasutus

Peromyscus ochraventer

Peromyscus pectoralis

Peromyscus pembertoni

Peromyscus perfulvus

Peromyscus polionotus

Peromyscus polius

Peromyscus pseudocrinitus

Peromyscus sagax

Peromyscus schmidlyi

Peromyscus sejugis

Peromyscus simulus

Peromyscus slevini

Peromyscus spicilegus

Peromyscus stephani

Peromyscus truei

Peromyscus winkelmann

Peromyscus yucatanicus

Peromyscus zarhynchus

Phenacomys intermedius

Phenacomys ungava

Podomys floridanus

Reithrodontomys bakeri

Reithrodontomys burti

Reithrodontomys chrysopsis

Reithrodontomys fulvescens

Reithrodontomys gracilis

Reithrodontomys hirsutus

Reithrodontomys humulis

Reithrodontomys megalotis

Reithrodontomys mexicanus

Reithrodontomys microdon

Naked-eared deermouse

Hooper's deermouse

Transvolcanic deermouse

San Lorenzo deermouse

Northwestern deermouse

White-footed deermouse

Broad-faced deermouse

Puebla deermouse

Black-wristed deermouse

Plateau deermouse

Black-eared deermouse

Black-tailed deermouse

Mesquite deermouse

Mexican deermouse

Northern rock deermouse

Brown belly deermouse

White-ankled deermouse

Pemberton's deermouse

Tawny deermouse

Oldfield deermouse

Chihuahuan deermouse

Coronados deermouse

Michoacán deermouse

Schmidly's deer mouse

Santa Cruz deermouse

Sinaloan deermouse

Catalina deermouse

Gleaning deermouse

San esteban deermouse

Pinyon deermouse

Coalcomán deermouse

Yucatán deermouse

Chiapan deermouse

Western heather vole

Eastern heather vole

Florida deermouse

Baker's harvest mouse

Sonoran harvest mouse

Volcano harvest mouse

Fulvous harvest mouse

Slender harvest mouse

Hairy harvest mouse

Eastern harvest mouse

Western harvest mouse

Mexican harvest mouse
Northern white-ankled deermouse ratón de ancas blancas norteño

Northern blackish deer mouse ratón negruzco del norte

Nimble-footed deermouse

Tres Marías Island deermouse

North american deermouse

Small-toothed harvest mouse ratón negruzco

ratón del algodón

ratón piñonero mexicano

ratón de la isla Ángel de la Guarda

ratón de Guatemala

ratón de orejas desnudas

ratón de Coahuila

ratón del Eje Volcánico

ratón de Isla San Lorenzo

ratón del Noroeste

ratón de patas blancas

ratón de patas agiles

ratón de Isla Tres Marías

ratón norteamericano/ratón de campo común

ratón de rostro ancho

ratón de Puebla

ratón de patas negras

ratón del altiplano

ratón de orejas negras

ratón de cola negra

ratón de los mezquites

ratón mexicano

ratón de las rocas del norte

ratón de vientre café

ratón de ancas blancas

ratón de la Isla San Pedro Nolasco

ratón leonado

ratón del sureste

ratón del Desierto Chihuahuense

ratón de Isla Coronados

ratón de Michoacán

ratón de la Sierra Madre Occidental

ratón de Isla Santa Cruz

ratón de Sinaloa

ratón de la Isla Catalina

ratón espiguero

ratón de Isla San Esteban

ratón piñonero del norte

ratón de Coalcomán

ratón de Yucatán

ratón de Chiapas

campañol del oeste

campañol del este

ratón de Florida

ratón cosechero guerrerense

ratón cosechero sonorense

ratón cosechero de los volcanes

ratón cosechero leonado

ratón cosechero esbelto

ratón cosechero peludo

ratón cosechero del este

ratón cosechero común

ratón cosechero mexicano

ratón cosechero de dientes pequeños
Reithrodontomys montanus Plains harvest mouse

Reithrodontomys raviventris Salt-marsh harvest mouse

Reithrodontomys spectabilis Cozumel harvest mouse

Reithrodontomys sumichrasti Sumichrast's harvest mouse

Reithrodontomys tenuirostris Narrow-nosed harvest mouse

Reithrodontomyszacatecae Zacatecan harvest mouse

Rheomys mexicanus

Mexican water mouse

Rheomys thomas

Scotinomys teguina

Sigmodon alleni

Sigmodon arizonae

Sigmodon fulviventer

Sigmodon hispidus

Sigmodon leucotis

Sigmodon mascotensis

Sigmodon ochrognathus

Sigmodon planifrons

Sigmodon toltecus

Sigmodon zanjonensis

Synaptomys borealis

Synaptomys cooperi

Tylomys bullaris

Tylomys nudicaudus

Tylomys tumbalensis

Xenomys nelsoni

Cuniculidae

Cuniculus paca

Dasyproctidae

Dasyprocta mexicana

Dasyprocta punctata

Thomas water mouse

Short-tailed singing mouse

Allen's cotton rat

Arizona cotton rat

Tawny-bellied cotton rat

Hispid cotton rat

White-eared cotton rat

West mexican cotton rat

Yellow-nosed cotton rat

Miahuatlán cotton rat

Toltec cotton rat

Montane cotton rat

Northern bog lemming

Southern bog lemming

Chiapan climbing rat

Peters's climbing rat

Tumbalá climbing rat

Magdalena woodrat

Lowland paca

Mexican agouti

Central american agouti

Erethizontidae

Erethizon dorsatum

Coendou mexicanus

Geomyidae

Cratogeomys castanops

Cratogeomys fulvescens

Cratogeomys fumosus

Cratogeomys goldmani

Cratogeomys merriami

Cratogeomys perotensis

Cratogeomys planiceps

Heterogeomys hispidus

Heterogeomys lanius

Geomys arenarius

Geomys attwateri

Geomys breviceps

Geomys bursarius

Geomys jugossicularis

Geomys knoxjonesi

Geomys lutescens

Geomys personatus

Geomys pinetis

Geomys streckeri

Geomys texensis

Geomys tropicalis

Oriental Basin pocket gopher

Smoky pocket gopher

Goldman's pocket gopher

Merriam's pocket gopher

Perote pocket gopher

Volcán de Toluca pocket gopher

Hispid pocket gopher

Big pocket gopher

Desert pocket gopher

Attwater's pocket gopher

Baird's pocket gopher

Plains pocket gopher

Hall's pocket gopher

Knox jones's pocket gopher

ratón cosechero de las planicies

ratón cosechero de la Bahía de San Francisco

ratón cosechero de Isla Cozume

ratón cosechero de montaña

ratón cosechero de Guatemala

ratón cosechero de Zacatecas

ratita nadadora mexicana

ratita nadadora de Chiapas

ratón pígmeo negro

rata matorralera del occidente

rata matorralera de Arizona

rata matorralera de vientre amarillo

rata cañera espinosa

rata matorralera orejas blancas

rata cañera del oeste

rata matorralera rostro amarillo

rata matorralera de Miahuatlán

rata cañera del Golfo

rata matorralera de las montañas

leming de pantano del norte

leming de pantano del sur

rata de árbol de Chiapas

rata de árbol común

rata de árbol de Tumbalá

rata de Magdalena

tepezcuintle/agutí manchado/cuautuza

agutí negro/guaqueque negro/serete

agutí centroamericano/guaqueque

centroamericano

North american porcupine puercoespín norteamericano

Mexican hairy dwarf porcupine puercoespín tropical/vistlacuache

Yellow-faced pocket gopher tuza cara amarilla 
Continue...

\begin{tabular}{|c|c|c|c|c|c|}
\hline Orthogeomys grandis & Giant pocket gopher & tuza gigante del pácifico & Dipodomys venustus & Narrow-faced kangaroo rat & rata canguro de rostro angosto \\
\hline Pappogeomys bulleri & Buller's pocket gopher & tuza jalisiense & Heteromys desmarestianus & Desmarest's spiny pocket mouse & ratón espinoso trópical \\
\hline Thomomys bottae & Botta's pocket gopher & tuza del norte & Heteromys gaumeri & Gaumer's spiny pocket mouse & rata de bolsas espinosa de Yucatán \\
\hline Thomomys atrovarius & Black-and-brown pocket gopher & tuza de Sinaloa & Heteromys goldmani & Goldmani's spiny pocket mouse & rata de bolsas espinosa de las tierras altas de \\
\hline Thomomys bulbivorus & Camas pocket gopher & tuza del Valle de Camas & & & Chiapas \\
\hline Thomomys clusius & Wyoming pocket gopher & tuza de Wyoming & Heteromys nelsoni & Nelson's spiny pocket mouse & rata de bolsas espinosa del sur Chiapas \\
\hline Thomomys fulvus & Fulvus pocket gopher & tuza del suroeste & Heteromys irroratus & Mexican spiny pocket mouse & rata de bolsas espinosa mexicano \\
\hline Thomomys idahoensis & Idaho pocket gopher & tuza de Idaho & Heteromys pictus & Painted spiny pocket mouse & rata de bolsas espinosa pintado \\
\hline Thomomys mazama & Western pocket gopher & tuza del oeste & Heteromys salvini & Salvin's spiny pocket mouse & rata de bolsas espinosa de Centroamerica \\
\hline Thomomys monticola & Mountain pocket gopher & tuza de las montañas & Heteromys spectabilis & Jaliscan spiny pocket mouse & rata de bolsas espinosa de Jalisco \\
\hline Thomomys nayarensis & Nayarit pocket gopher & tuza del Nayar & Microdipodops megacephalus & Dark kangaroo mouse & ratón canguro oscuro \\
\hline Thomomys nigricans & Baja California pocket gopher & tuza de Baja California & Microdipodops pallidus & Pale kangaroo mouse & ratón canguro pálido \\
\hline Thomomys sheldoni & Sierra Madre Occidental pocket & tuza de pequeña de la Sierra Madre Occidental & Perognathus alticolus & White-eared pocket mouse & ratón de bolsas de orejas blancas \\
\hline & gopher & & Perognathus amplus & Arizona pocket mouse & ratón de bolsas de Arizona \\
\hline Thomomys talpoides & Northern pocket gopher & tuza del norte & Perognathus fasciatus & Olive-backed pocket mouse & ratón de bolsas verdoso \\
\hline Thomomys townsendii & Townsend's pocket gopher & tuza de las montañas del oeste & Perognathus flavescens & Plains pocket mouse & ratón de bolsas de las planicies \\
\hline Thomomys umbrinus & Southern pocket gopher & tuza mexicana & Perognathus flavus & Silky pocket mouse & ratón de bolsas sedoso \\
\hline Zygogeomys trichopus & Michoacan pocket gopher & tuza michoacana & Perognathus inornatus & San joaquin pocket mouse & ratón de bolsas del Valle de San Joaquín \\
\hline Heteromyidae & & & Perognathus longimembris & Little pocket mouse & ratón de bolsas minúsculo \\
\hline Chaetodipus ammophilus & Dalquest's pocket mouse & ratón de bolsas del Sur de Baja California & Perognathus merriami & Merriam's pocket mouse & ratón de bolsas del este \\
\hline Chaetodipus arenarius & Little desert pocket mouse & ratón de bolsas abazones de Baja California & Perognathus parvus & Great basin pocket mouse & ratón de bolsas de la Great Basin \\
\hline Chaetodipus artus & Narrow-skulled pocket mouse & ratón de bolsas de cara delgada & Sciuridae & & \\
\hline Chaetodipus baileyi & Bailey's pocket mouse & ratón de bolsas gigante & Ammospermophilus harrisii & Harris's antelope squirrel & ardilla listada Sonorense \\
\hline Chaetodipus californicus & California pocket mouse & ratón de bolsas de California & Ammospermophilus interpres & Texas antelope squirrel & ardilla listada Texana \\
\hline Chaetodipus eremicus & Chihuahuan pocket mouse & ratón de bolsas Chihuahuense & Ammospermophilusleucurus & White-tailed antelope squirrel & juancito/ardilla listada coliblanca \\
\hline Chaetodipus fallax & San diego pocket mouse & ratón de bolsas San Diego & Ammospermophilus nelsoni & Nelson's antelope squirrel & ardilla listada del Valle de San Joaquin \\
\hline Chaetodipus formosus & Long-tailed pocket mouse & ratón de bolsas de cola larga & Callospermophilus lateralis & Golden-mantled ground squirrel & ardilla de montaña dorada \\
\hline Chaetodipus goldmani & Goldman's pocket mouse & ratón de bolsas Sonorense & Callospermophilus madrensis & Sierra Madre ground squirrel & ardilla de montaña de la Sierra Madre \\
\hline Chaetodipus hispidus & Hispid pocket mouse & ratón de bolsas de los pastizales & Callospermophilus saturatus & Cascade ground squirrel & ardilla de montaña dorada \\
\hline Chaetodipus intermedius & Rock pocket mouse & ratón de bolsas de las rocas & Cynomys gunnisoni & Gunnison's prairie dog & perrito de las praderas de la planicie de Colorado \\
\hline Chaetodipus lineatus & Lined pocket mouse & ratón de bolsas Potosino & Cynomys leucurus & White-tailed prairie dog & perrito de las praderas coliblanca \\
\hline Chaetodipus nelsoni & Nelson's pocket mouse & ratón de bolsas del altiplano & Cynomys ludovicianus & Black-tailed prairie dog & perrito de las praderas colinegra \\
\hline Chaetodipus penicillatus & Desert pocket mouse & ratón de bolsas del desierto & Cynomys mexicanus & Mexican prairie dog & perrito de las praderas mexicano \\
\hline Chaetodipus pernix & Sinaloan pocket mouse & ratón de bolsas de Sinaloa & Cynomys parvidens & Utah prairie dog & perrito de las praderas de Utah \\
\hline Chaetodipus rudinoris & Baja california pocket mouse & ratón de bolsas de Baja California & Glaucomys sabrinus & Northern flying squirrel & ardilla voladora del norte \\
\hline Chaetodipus siccus & Cerralvo island pocket mouse & ratón de bolsas de Isla Cerralvo & Glaucomys volans & Southern flying squirrel & ardilla voladora del sur \\
\hline Chaetodipus spinatus & Spiny pocket mouse & ratón de bolsas espinoso & Ictidomys mexicanus & Mexican ground squirrel & motocle/ardilla manchada mexicana \\
\hline Dipodomys agilis & Agile kangaroo rat & rata canguro del Sur de California & Ictidomys parvidens & Texas ground squirrel & ardilla manchada texana \\
\hline Dipodomys californicus & California kangaroo rat & rata canguro de California & Ictidomys tridecemlineatus & Thirteen-lined ground squirrel & ardilla manchada de trecelíneas \\
\hline Dipodomys compactus & Gulf coast kangaroo rat & rata canguro de la costa del Golfo & Marmota broweri & Alaska marmot & marmota de Alaska \\
\hline Dipodomys deserti & Desert kangaroo rat & rata canguro del desierto & Marmota caligata & Hoary marmot & marmota canosa \\
\hline Dipodomys elator & Texas kangaroo rat & rata canguro de Texas & Marmota flaviventris & Yellow-bellied marmot & marmota de vientre amarillo \\
\hline Dipodomys gravipes & San Quintin kangaroo rat & rata canguro de San Quintín & Marmota monax & Woodchuck & marmota americana \\
\hline Dipodomys heermanni & Heermann's Kangaroo Rat & rata canguro de la Bahía el Morro & Marmota olympus & Olympic marmot & marmota de la península de Olimpia \\
\hline Dipodomys ingens & Giant kangaroo rat & rata canguro gigante & Marmota vancouverensis & Vancouver island marmot & marmota de Isla Vancouver \\
\hline Dipodomys merriami & Merriam's kangaroo rat & rata canguro mexicana & Notocitellus adocetus & Tropical ground squirrel & cuinique, juancito del balsas \\
\hline Dipodomys microps & Chisel-toothed kangaroo rat & rata canguro con incisivos de cincel & Notocitellus annulatus & Ring-tailed ground squirrel & ardilla de tierra cola anillada \\
\hline Dipodomys nelsoni & Nelson's kangaroo rat & rata canguro del altiplano norte & Otospermophilus beecheyi & California ground squirrel & ardillón de California \\
\hline Dipodomys nitratoides & San Joaquin valley kangaroo rat & rata canguro del Valle de San Joaquín & Otospermophilus douglasii & Douglas ground squirrel & ardillón de California del norte \\
\hline Dipodomys ordii & Ord's kangaroo rat & rata canguro común & Otospermophilus variegatus & Rock squirrel & ardillón común \\
\hline Dipodomys ornatus & Plateau kangaroo rat & rata canguro coliblanca del norte & Poliocitellus franklinii & Franklin's ground squirrel & ardilla de tierra gris \\
\hline Dipodomys panamintinus & Panamint kangaroo rat & rata canguro del Valle de Panamint & Sciurus aberti & Abert's squirrel & ardilla orejas peludas \\
\hline Dipodomys phillipsii & Phillips's kangaroo rat & rata canguro coliblanca del sur & Sciurus alleni & Allen's squirrel & ardilla de la Sierra Madre Oriental \\
\hline Dipodomys simulans & Dulzura kangaroo rat & rata canguro de Baja California & Sciurus arizonensis & Arizona gray squirrel & ardilla de Arizona \\
\hline Dipodomys spectabilis & Banner-tailed kangaroo rat & rata canguro de cola blanca & Sciurus aureogaster & Red-bellied squirrel & ardilla de vientre rojo/ardilla gris mexicana \\
\hline Dipodomys stephensi & Stephens'kangaroo rat & rata canguro del Valle Moreno & Sciurus carolinensis & Eastern gray squirrel & ardilla gris del este \\
\hline
\end{tabular}


Continue...

Sciurus colliaei

Sciurus deppei

Sciurus griseus

Sciurus nayaritensis

Sciurus niger

Sciurus oculatus

Sciurus variegatoides

Sciurus yucatanensis

Tamias alpinus

Tamias amoenus

Tamias bulleri

Tamias canipes

Tamias cinereicollis

Tamias dorsalis

Tamias durangae

Tamias merriami

Tamias minimus

Tamias obscurus

Tamias ochrogenys

Tamias palmeri

Tamias panamintinus

Tamias quadrimaculatus

Tamias quadrivittatus

Tamias ruficaudus

Tamias rufus

Tamias senex

Tamias siskiyou

Tamias solivagus

Tamias sonomae

Tamias speciosus

Tamias striatus

Tamias townsendii

Tamias umbrinus

Tamiasciurus douglasii

Tamiasciurus hudsonicus

Tamiasciurus mearnsi

Urocitellus armatus

Urocitellus beldingi

Urocitellus brunneus

Urocitellus canus

Urocitellus columbianus

Urocitellus elegans

Urocitellus mollis

Urocitellus parryii

Urocitellus richardsonii

Urocitellus townsendii

Urocitellus washingtoni

Xerospermophilusmohavensis

Xerospermophilus perotensis

Xerospermophilus spilosoma

Xerospermophilustereticaudus

Zapodidae

Napaeozapus insignis

Zapus hudsonius

Zapus princeps

Zapus trinotatus
Collie's squirrel

Deppe's squirrel

Western gray squirrel

Mexican fox squirrel

Eastern fox squirrel

Peters's squirrel

Variegated squirrel

Yucatan squirrel

Alpine chipmunk

Yellow-pine chipmunk

Buller's chipmunk

Gray-footed chipmunk

Gray-collared chipmunk

Cliff chipmunk

Durango chipmunk

Merriam's chipmunk

Least chipmunk

California chipmunk

Yellow-cheeked chipmunk

Palmer's chipmunk

Panamint chipmunk

Long-eared chipmunk

Colorado chipmunk

Red-tailed chipmunk

Hopi chipmunk

Allen's chipmunk

Siskiyou chipmunk

Howell's chipmunks

Sonoma chipmunk

Lodgepole chipmunk

Eastern chipmunk

Townsend's chipmunk

Uinta chipmunk

Douglas'squirrel

Red squirrel

Mearns's squirrel

Uinta ground squirrel

Belding's ground squirrel

Idaho ground squirrel

Merriam's ground squirrel

Columbian ground squirrel

Wyoming ground squirrel

Piute ground squirrel

Arctic ground squirrel

Richardson's ground squirrel

Townsend's ground squirrel

Washington ground squirrel

Mohave ground squirrel

Perote ground squirrel

Spotted ground squirrel

Round-tailed ground squirrel

Woodland jumping mouse

Meadow jumping mouse

Western jumping mouse

Pacific jumping mouse ardilla de la Sierra Madre Occidental

ardilla tropical

ardilla gris del oeste

ardilla de Nayarit

ardilla zorra del este

ardilla mexicana

ardilla Centroamérica

ardilla Yucateca

chichimoco alpino

chichimoco del pino ponderosa

chichimoco de Zacatecas

chichimoco de patas grises

chichimoco de collar gris

chichimoco de los acantilados

chichimoco de Durango

chichimoco del sur de California

chichimoco pigmeo

chichimoco de California

chichimoco de cachetes amarillos

chichimoco de las Montañas Spring

chichimoco del Valle Panamint

chichimoco de orejas grandes

chichimoco de Colorado

chichimoco de cola roja

chichimoco de los Hopi

chichimoco de la Sierra Nevada

chichimoco de las montañas Siskiyou

chichimoco de Coahuila

chichimoco de Sonoma

chichimoco del pino

chichimoco de este

chichimoco del noroeste

chichimoco común

ardilla de la costa oeste

ardilla roja

ardilla de San Pedro Mártir

ardilla de tierra de las montañas Uinta

ardilla de tierra del oeste

ardilla de tierra de Idaho

ardilla de tierra canosa

ardilla de tierra de la Columbia Británica

ardilla de tierra de Wyoming

ardilla de tierra del pueblo Piute

ardilla de tierra del ártico

ardilla de tierra de las praderas

ardilla de tierra de las planicies

ardilla de tierra de Washington

ardilla de tierra de desierto de Mohave

ardilla de tierra de Perote

ardilla de tierra manchada, juancito manchado

ardilla de tierra cola redonda

ratón saltarín de los boques

ratón saltarín de las praderas

ratón saltarín del oeste

ratón saltarín del Pacífico

SIRENIA

Trichechidae

Trichechus manatus West indian manatee manatí/vaca marina
Soricomorpha

\section{Soricidae}

Blarina brevicauda

Blarina carolinensis

Blarina hylophaga

Blarina peninsulae

Cryptotis alticola

Cryptotis goldmani

Cryptotis goodwini

Cryptotis griseoventris

Cryptotis lacandonensis

Cryptotis magnus

Cryptotis mayensis

Cryptotis merriami

Cryptotis mexicanus

Cryptotis nelsoni

Cryptotis obscurus

Cryptotis parvus

Cryptotis peregrina

Cryptotis phillipsii

Cryptotis tropicalis

Megasorex gigas

Notiosorex cockrumi

Notiosorex crawfordi

Notiosorex evotis

Notiosorex villai

Sorex alaskanus

Sorex albibarbis

Sorex arcticus

Sorex arizonae

Sorex bairdii

Sorex bendirii

Sorex cinereus

Sorex dispar

Sorex emarginatus

Sorex fumeus

Sorex gaspensis

Sorex haydeni

Sorex hoyi

Sorex ixtlanensis

Sorex jacksoni

Sorex longirostris

Sorex lyelli

Sorex macrodon

Sorex maritimensis

Sorex mediopua

Sorex merriami

Sorex milleri

Sorex minutissimus

Sorex monticola

Sorexnanus

Sorex navigator
Northern short-tailed shrew

Southern short-tailed shrew

Elliot's short-tailed shrew

Eeverglades short-tailed shrew

Central mexican broad-clawed

shrew

Goldman's broad-clawed shrew

musaraña tropical de la Sierra Madre del Sur

oodwin's broad-clawed shrew musaraña tropical de Chiapas

Guatemalan broad-clawed shrew musaraña tropical de Guatemala

Lacandona small-eared shrew musaraña tropical de la Lacandona

Big mexican small-eared shrew musaraña tropical gigante

Yucatan small-eared shrew musaraña tropical Maya

Merriam's small-eared shrew musaraña tropical de tierras altas

Mexican small-eared shrew

musaraña tropical mexicana

Nelson's small-eared shrew

musaraña tropical de Catemaco

Grizzled mexican small-eared

shrew

North american least shrew

Oaxacan broad-clawed shrew

Phillips'small-eared shrew

Tropical small-eared shrew

Mexican shrew

Cockrum's gray shrew

Crawford's gray shrew

Large-eared gray shrew

Villa's gray shrew

Glacier bay water shrew

Eastern water shrew

Arctic shrew

Arizona shrew

Baird's shrew

Marsh shrew

Cinereus shrew

Long-tailed shrew

Zacatecas shrew

Smoky shrew

Gaspé shrew

Prairie shrew

American pygmy shrew

Ixtlan shrew

St. Lawrence island shrew

Southeastern shrew

Mt. Lyell shrew

Large-toothed shrew

Maritime shrew

Jalisco shrew

Merriam's shrew

Carmen mountain shrew

Eurasian least shrew

Dusky shrew

Dwarf shrew

Western water shrew

musaraña tropical de Hidalgo

musaraña tropical de Norte América

musaraña tropical de Oaxaca

musaraña tropical la sierra sur de Oaxaca

musaraña de tropica

musaraña gigante

musaraña del desierto de Arizona

musaraña del desierto común

musaraña del desierto del Pacífico 
Continue...

\begin{tabular}{|c|c|c|}
\hline Sorex neomexicanus & New Mexico shrew & musaraña de Nuevo México \\
\hline Sorex oreopolus & Mexican long-tailed shrew & musaraña mexicana \\
\hline Sorex orizabae & Orizaba long-tailed shrew & musaraña de Orizaba \\
\hline Sorex ornatus & Ornate shrew & musaraña de las californias \\
\hline Sorex pacificus & Pacific shrew & musaraña del Pacífico \\
\hline Sorex palustris & American water shrew & musaraña de agua americana \\
\hline Sorex preblei & Preble's shrew & musaraña del noroeste \\
\hline Sorex pribilofensis & Pribilof island shrew & musaraña de la isla Pribilof \\
\hline Sorex rohweri & Olympic shrew & musaraña de la peninsula Olimpia \\
\hline Sorex salvini & Salvin's shrew & musaraña del sureste \\
\hline Sorex saussurei & Saussure's shrew & musaraña común de montaña \\
\hline Sorex sclateri & Sclater's shrew & musaraña selvática \\
\hline Sorex sonomae & Fog shrew & musaraña de la niebla \\
\hline Sorex stizodon & San Cristobal shrew & musaraña de San Cristóbal de las Casas \\
\hline Sorex tenellus & Inyo shrew & musaraña de las montañas Inyo \\
\hline Sorex trowbridgii & Trowbridge's shrew & musaraña de la costa oeste \\
\hline Sorex tundrensis & Tundra shrew & musaraña de la tundra \\
\hline Sorex ugyunak & Barren ground shrew & musaraña del Ártico \\
\hline Sorex vagrans & Vagrant shrew & musaraña común del oeste \\
\hline Sorex ventralis & Chestnut-bellied shrew & musaraña de vientre castaño \\
\hline Sorex veraecrucis & Veracruz shrew & musaraña de Veracruz \\
\hline Sorex veraepacis & Verapaz shrew & musaraña de las tierras altas del sur \\
\hline \multicolumn{3}{|l|}{ Talpidae } \\
\hline Condylura cristata & Star-nosed mole & topo nariz de estrella \\
\hline Neurotrichus gibbsii & Shrew-mole & topo musaraña \\
\hline Parascalops breweri & Hairy-tailed mole & topo de cola peluda \\
\hline Scapanus anthonyi & Anthony's mexican mole & topo de San Pedro Mártir/topo de manoplas \\
\hline Scalopus aquaticus & Eastern mole & topo de la costa este \\
\hline Scapanus latimanus & Broad-footed mole & topo de la costa oeste \\
\hline Scapanus orarius & Coast mole & topo de la costa \\
\hline Scapanus townsendii & Townsend's mole & topo de la costa noroeste \\
\hline
\end{tabular}

\section{Acknowledgments}

We are grateful to colleagues, students and friends who have shared common names in Spanish and English with us over the years. G. Ameneyro discussed some ideas expressed in this work. Two anonymous reviewers improved the quality of the writing. María Elena Sánchez-Salazar translated the manuscript into English.

\section{Literatura Citada}

AlLEN, H. 1894. A monograph of the bats of North America. Bulletin of the United States National Museum 43:1-98.

Álvarez-Castañeda, S. T., and T. Álvarez Solorzano. 1996. Etimologías de los géneros de mamíferos mexicanos. Ciencia 47:39-49.

Álvarez-Castañeda, S. T., T. Álvarez, and N. González-Ruiz. 2015. Guía para la identificación de los mamíferos de México en campo y laboratorio. Centro de Investigaciones Biológicas del Noroeste, S. C., and Asociación Mexicana de Mastozoología, A. C. Guadalajara, México.

Álvarez-Castañeda, S. T., T. Álvarez, and N. González-Ruiz. 2017. Keys for identifying Mexican Mammals. The Johns Hopkins University Press. Baltimore, U. S. A.

Álvarez, T., And M. GonzÁlez E. 1987. Fauna. Atlas Cultural de México. Secretaría de Educación Pública, Instituto Nacional de Antropología e Historia, Grupo Editorial Planeta 9:1-187.
Anderson, S. 1972. Mammals of Chihuahua, taxonomy and distribution. Bulletin of the American Museum of Natural History 148: 149-410.

Aranda, M. 2000. Huellas y otros rastros de los mamíferos grandes y medianos de México. CONABIO e Instituto de Ecología, A. C. Ciudad de México, México.

ARITA, T., H., AND S. R. HumphreY. 1988. Revisión taxonómica de los murciélagos magueyeros del género Leptonycteris (Chiroptera: Phyllostomidae). Acta Zoológica Mexicana (n. s.) 29:1-60.

Baker, R. J., L. C. Bradley, R. D. Bradley, J. W. Dragoo, M. D. Engstrom, R. S. Hoffmann, C. A. Jones, D. W. Rice, AND C. Jones. 2003. Revised checklist of North American mammals north of México. Occasional Papers, Museum Texas Tech University 229:1-23.

Ceballos, G., AND G. Oliva (Coords.). 2005. Los mamíferos silvestres de México. Comisión Nacional para el Conocimiento y Uso de la Biodiversidad y Fondo de Cultura Económica. Ciudad de México, México.

Ceballos, G., and C. L. Galindo. 1984. Mamíferos silvestres de la Cuenca de México. Editorial Limusa. Ciudad de México, México.

ClutTon-Brock, J. 2015. Naming thescale of nature. Pp. 171 in Taxonomic tapestries: the threads of evolutionary, behavioural and conservation research (Behie A. M., and M. F. Oxenham, eds.). Australian National University Press, Australian.

Escalante, P., A. M. SADA, AND J. G. Robles. 2014. Listado de nombres comunes de las aves de México. Universidad Nacional Autónoma de México y Sociedad para el Estudio y Conservación de las Aves en México, A. C. Ciudad de México, México.

Gallo-Reynoso, J. P. 1985. Nombres científicos y comunes de los mamíferos marinos de México. Anales del Instituto de Biología, Serie Zoología 56:1043-1056.

Gaumer, G. F. 1917. Monografía de los mamíferos de Yucatán. Departamento. de Talleres Gráficos de la Secretaría de Fomento. Ciudad de México, México.

González-Christen, A (COORD). 2010. Los mamíferos de Veracruz. Guía ilustrada. Colección la Ciencia en Veracruz, Consejo Veracruzano de Investigación Científica y Desarrollo Tecnológico, México.

HaLL, E. R. 1981. The mammals of North America. John Wiley and Sons.

Hall, E. R., AND W. W. Dalquest. 1963. The mammals of Veracruz. University of Kansas Publications, Museum of Natural History 14:165-362.

Hilton-TAYloR, C. (compiler). 2000. 2000 IUCN Red List of Threatened Species. International Union for Conservation of Nature, Gland and Cambridge, Switzerland and United Kindom.

ICZN. 1999. International Code of Zoological Nomenclature. Fourth edition. The International Trust for Zoological Nomenclature. London, United Kindom.

JACKSON, S. M., AND C. P. GROVES. 2015. Taxonomy of Australian Mammals. CSIRO Publishing. Melbourne, Australia.

Jones, J. K., JR., D. C. CARTER, AND H. H. Genoways. 1973. Checklist of North American mammals north of Mexico. Occasional Papers, Museum of Texas Tech University 12:1-14.

KERR, R. 1792. The Animal Kingdom, or zoological system, of the celebrated Sir Charles Linnæus: Class I. Mammalia. Edinburgh: A. Strahan, and London: T. Cadell, and Edinburgh: W. Creech. 
León-Paniagua, L., A. Navarro-Sigüenza, B. E. Hernández-Baños, and J. C. Morales. 2007. Diversification of the arboreal mice of the genus Habromys (Rodentia: Cricetidae: Neotominae) in the Mesoamerican highlands. Molecular Phylogenetics and Evolution 42:653-664.

LinNAEUS, C. 1758. Systema naturae, 10th edn, vol. 1. Stockholm: L. Salvii.

MeRRIAM, C. H. 1918. Review of the grizzly and big brown bears of North America (genus Ursus), with description of a new genus Vetularctos. North American Fauna 41:1-136.

Mones, A., And U. K. Mones. 1991. Comentarios sobre el uso de los nombres vulgares en zoología. Anales del Instituto de Biología, Serie Zoología 62:249-252.

Porter, C. A., N. E. Beasley, N. Ordóñez-Garza, L. L. Lindsey, D. S. Rogers, N. Lewis-Rogers, J. W. Sites, and R. D. Bradley. 2017. A new species of big-eared climbing rat, genus Ototylomys (Cricetidae: Tylomyinae), from Chiapas, Mexico. Journal of Mammalogy 98:1310-1329.

RamíreZ-Pulido, J., R. LóPEZ-Wilchis, C. MüdesPaCher, AND I. LIRA. 1983. Lista y bibliografía reciente de los mamíferos de México. Universidad Autónoma Metropolitana-Iztapalapa y Editorial Contraste. Ciudad de México, México.

ReID, F. 2006. Peterson field guide to mammals of North America. Fourth edition. Houghton Mifflin. New York, U. S. A.

Sánchez-Hernández, C., M. L. Romero-Almaraz, G. D. Schnell, M. L. Kennedy, T. L. Best, R. D. Owen, and S. B. González-Pérez. 2016. Bats of Colima, Mexico. University of Oklahoma Press. Norman, Oklahoma.

SÁnCHEZ, O. 1985. Los mamíferos en las culturas antiguas de México. Zacatuche 2:2-12.

SEMARNAT (Secretaría de Medio Ambiente y Recursos Naturales). 2002. Norma Oficial Mexicana NOM-059- ECOL2001. Protección ambiental-Especies nativas de México de flora y fauna silvestres-Categorías de riesgo y especificaciones para su inclusión, exclusión o cambio, y lista de especies en riesgo. Diario Oficial de la Federación, 6 de marzo de 2002, Segunda Sección. Ciudad de México, México.

SEMARNAT (Secretaría de Medio Ambiente y Recursos Naturales). 2010. Norma Oficial Mexicana NOM-059SEMARNAT-2010. Protección ambiental, especies nativas de flora y fauna silvestres de México, categorías de riesgo y especificaciones para su inclusión, exclusión o cambio, y lista de especies en riesgo. Diario Oficial de la Federación, 30 de diciembre de 2010, Segunda Sección. Ciudad de México, México.

Simpson, G. G. 1941. Vernacular Names of South American Mammals. Journal of Mammalogy 22:1-17.

Verts, B. J., and L. Carraway. 1998. Land Mammals of Oregon. University of California Press. Berkeley, U. S. A.

VilLA-R., B. 1966. Los murciélagos de México. Instituto de Biología, Universidad Nacional Autónoma de México. Ciudad de México, México.

Villa Ramírez, B., and F. A. Cervantes. 2003. Los mamíferos de México. Grupo Editorial Iberoamérica e Instituto de Biología, Universidad Nacional Autónoma de México. Ciudad de México, México.

Wilson, D. E., AND D. M. ReEDeR (eds.). 2005. Mammal species of the world. A taxonomic and geographic reference, Third edition. The Johns Hopkins University Press. Baltimore, U. S. A.
WILSON D. E., AND F. R. ColE. 2000. Common names of mammals of the world. Smithsonian Institution Press. Washington, U.S. A. WILson D. E., AND S. Ruff (EDS.). 1999. The Smithsonian book of North American mammals. Smithsonian Institution Press. Washington, U.S. A.

Associated editor: Rafael Reyna

Submitted: December 22, 2017; Reviewed: January 18, 2018;

Accepted: January 18, 2018; Published on line: January 26, 2018. 